\title{
DNA Double Helices for Single Molecule Electronics
}

\author{
A. V. Malyshev \\ GISC, Departamento de Física de Materiales, Universidad Complutense, Madrid 28040, Spain \\ and A. F. Ioffe Physico-Technical Institute, 194021 St. Petersburg, Russia
}

(Received 13 December 2006; published 28 February 2007)

\begin{abstract}
The combination of self-assembly and electronic properties as well as its true nanoscale dimensions make DNA a promising candidate for a building block of single molecule electronics. We argue that the intrinsic double helix conformation of the DNA strands provides a possibility to drive the electric current through the DNA by the perpendicular electric (gating) field. The transistor effect in the poly $(\mathrm{G})-\operatorname{poly}(\mathrm{C})$ synthetic DNA is demonstrated within a simple model approach. We put forward experimental setups to observe the predicted effect and discuss possible device applications of DNA. In particular, we propose a design of the single molecule analog of the Esaki diode.
\end{abstract}

DOI: 10.1103/PhysRevLett.98.096801

PACS numbers: 85.35. $-\mathrm{p}, 85.30 . \mathrm{Mn}, 85.30 . \mathrm{Tv}, 87.14 . \mathrm{Gg}$

The controversial question of charge transport in DNA molecules has been attracting a great deal of attention recently (see Refs. [1-3] for an overview). The interest in DNA transport properties is at least twofold: on the one hand, the charge migration is believed to be important for the radiation damage repair [4] and, on the other, DNA double helices are expected to be particularly useful for molecular electronics [3,5-7]. While random base sequences are relevant for biological samples, artificially created periodic DNA molecules [8], such as the poly(A)-poly(T) or poly $(\mathrm{G})$-poly $(\mathrm{C})$, are probably the best candidates for novel device applications. The electrical transport through dry and wet DNA has been extensively studied both theoretically and experimentally and a variety of results has emerged: DNA has been reported to demonstrate proximity-induced superconducting [9], metallic [10-13], semiconducting [14-18], and insulating [19,20] behavior. Contact related effects, the impact of the environment, and the DNA base pair sequence lead to such diversity of results. According to both theory and experiment, the dry poly $(\mathrm{G})-\operatorname{poly}(\mathrm{C})$ synthetic DNA is a semiconductor: theoretical ab initio calculations predict a wide-band-gap semiconductor behavior (see, e.g., Ref. [21]) while experimental measurements reveal about $2 \mathrm{~V}$ voltage gap at low temperature [14].

Many effects useful for molecular device applications have been reported: rectification, the Kondo effect, the Coulomb blockade, etc. (see Ref. [7] for a recent overview). In this contribution, it is demonstrated for the first time that the intrinsic helix conformation of the DNA strands determines the transport properties of gated DNA molecules. In particular, we show that the electric current through the double helix DNA (in the base stacking direction) can be driven by the perpendicular gating field. We put forward new experimental setups to reveal the predicted effect and discuss possible applications of the DNA. In particular, we propose a design of the single molecule analog of the Esaki diode.
Two approaches are widely used to describe the DNA: $a b$ initio calculations [21-28] and model-based Hamiltonians [29-44]. The former can provide a detailed description but is currently limited to relatively short molecules (typically of the order of 10 base pairs long). The latter is much less detailed but allows for addressing systems of realistic length. The model-based approach can play an important complementary role because it grasps usually the underlying physics. Often, it yields quite satisfactory quantitative results as well.

Here, we focus on qualitative properties of the DNA and use, therefore, the effective Hamiltonian approach. A variety of models and parameter sets are being discussed (see Refs. [42,43] and references therein). In order to address intrinsic properties of DNA, we do not consider any environment or complex contact related effects and keep the formalism as simple as possible. Hence, we adopt the minimum tight-binding ladder model that accounts for the double-stranded structure of DNA. The ladder model was introduced in Ref. [29] and has widely been used since then (see Refs. [36,37,43] and references therein). The Hamiltonian of the model reads:

$$
\begin{aligned}
& \sum_{s, n}\left(\varepsilon_{s n}|s n\rangle\langle s n|-t| s n+1\rangle\langle s n|+\text { H.c. }-\tau|-s n\rangle\langle s n|\right) \\
& \quad+\sum_{s, k}\left(\varepsilon_{s M}|s k\rangle\left\langle s k\left|-t_{M}\right| s k+1\right\rangle\langle s k|+\text { H.c. }\right) \\
& \quad-\sum_{s}\left(\Gamma_{s l}|s 0\rangle\left\langle s 1\left|+\Gamma_{s r}\right| s N+1\right\rangle\langle s N|+\text { H.c. }\right),
\end{aligned}
$$

where the first term is the Hamiltonian of the $N$-base-pair DNA: $\varepsilon_{s n}$ are on-site energies of base molecules with index $n \in[1, N]$ labeling a pair and index $s= \pm 1$ labeling a strand, $t$ and $\tau$ are interbase hoppings parallel and perpendicular to the base stacking direction, respectively. The second term describes semi-infinite source $(k<1)$ and drain $(k>N)$ metallic leads with $\varepsilon_{s M}=E_{F}$ and $t_{M}=4 t$ [43], while the third is the DNA-contact coupling term with 
$\Gamma_{s l}=\Gamma_{s r}=t[33,43] .\langle s n|$ and $|s n\rangle$ are bra and ket vectors of an electron at site $n$ of the strand $s$.

Here, we extend the traditional ladder model (which neglects the helix geometry of the strands) to the case when a molecule is subjected to the perpendicular electric field and the helix conformation of the strands becomes important. The B form of the DNA with the 10-base-pairs full-twist period will be considered. Neglecting the difference between major and minor grooves we set the on-site energies $\varepsilon_{s n}$ as follows:

$$
\varepsilon_{s n}=\varepsilon_{s n}^{(0)}+e E_{n} s r \cos \left(\frac{2 \pi n}{10}+\varphi_{0}\right),
$$

where $\varepsilon_{s n}^{(0)}$ is site energy of the $s n$th base molecule at zero field, $E_{n}$ is the perpendicular gating field (for simplicity, let it be homogeneous: $E_{n}=E_{0}$ ), and $r \sim 1 \mathrm{~nm}$ is the strand radius. Hereafter, we use the notation $V_{g}=2 E_{0} r$ for the gate voltage drop across the double helix. The phase $\varphi_{0}$ that determines the orientation of the molecule with respect to the field is set to 0 from now on.

Equation (1) demonstrates that the perpendicular electric field results in the harmonic modulation of the potential along the helical strands. The modulation changes the electronic structure of the DNA and turns up to be crucial for transport properties as we show below. Moreover, the amplitude of the modulation can be controlled by the gating field, providing a mechanism to alter the fundamental properties of the system.

Throughout the Letter, the electron transport through a 31-base-pair poly $(\mathrm{G})$-poly (C) DNA molecule is addressed with the following lowest unoccupied molecular orbital (LUMO) state on-site energies which are used as a starting point: $\quad \varepsilon_{-1 n}^{(0)}=\varepsilon_{G}=1.14 \mathrm{eV}, \quad \varepsilon_{+1 n}^{(0)}=\varepsilon_{C}=-1.06 \mathrm{eV}$ [28]. Hopping integrals $t$ and $\tau$ are not considered as bare tight-binding parameters, rather as effective ones [43], and are adjusted to reproduce the voltage gap of about $2 \mathrm{~V}$, which was observed in experiments on the dry poly $(\mathrm{G})$-poly(C) DNA $[14,18]: \quad t=0.27 \mathrm{eV}, \quad \tau=$ $0.25 \mathrm{eV}$. These values are within reasonable parameter intervals [44]. Using the transmitting quantum boundary method (see Refs. [45,46] and references therein), we obtained the transmission coefficient of the system, $T\left(V_{g}, E\right)$, and calculated the current-voltage characteristics within the scattering formalism [47]:

$$
I=\frac{2 e}{h} \int T\left(V_{g}, E\right)\left[f_{s}\left(E, V_{\mathrm{sd}}\right)-f_{d}\left(E, V_{\mathrm{sd}}\right)\right] d E,
$$

where $f_{s, d}\left(E, V_{\mathrm{sd}}\right)=\left(1+e^{\left(E_{F} \pm e V_{\mathrm{sd}} / 2-E\right) / k T}\right)^{-1}$ are the Fermi functions of source and drain contacts, $V_{\text {sd }}$ is the source-drain voltage drop, and $E_{F}$ is the Fermi energy at equilibrium taken to be in the middle of the DNA band gap, as for Au contacts [48]. The temperature $T$ is set to $4 \mathrm{~K}$.

Figure 1 shows current-voltage characteristics and transmission coefficients of the poly $(\mathrm{G})$-poly $(\mathrm{C})$ DNA molecule at different gate voltages. In all cases the system behaves as

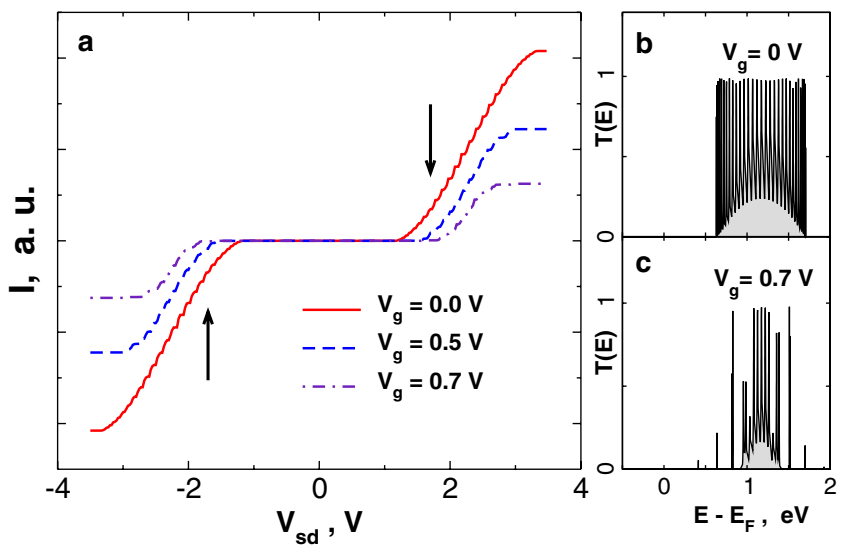

FIG. 1 (color online). (a) Current-voltage characteristics of the DNA at different gate voltages $V_{g}$. (b) and (c) transmission coefficient for $V_{g}=0$ and $V_{g}=0.7 \mathrm{~V}$.

a semiconductor with the voltage gap that varies with the gating field. Thus, within a range of source-drain voltages [in the vicinity of those indicated by vertical arrows in Fig. 1(a)], the system can be either conducting or insulating, depending on the field.

Figure 2 demonstrates current-voltage characteristics within a wide range of source-drain and gate voltages. Semiconducting behavior can be observed for $\left|V_{g}\right| \leq$ $0.7 \mathrm{~V}$ as well as a strong gating effect for $\left|V_{\text {sd }}\right| \geq 1.2 \mathrm{~V}$. To illustrate the gating effect, we plot in Fig. 3 dependencies of the current on the gate voltage drop for several fixed values of the source-drain voltage $V_{\mathrm{sd}}$. For all values of $V_{\mathrm{sd}}$, a typical hatlike $I-V_{g}$ characteristic is observed. Strong dependence of the source-drain current on the gate voltage suggests the usage of the gated double helix DNA as a field-effect transistor.

To perform conductance measurements, a linear DNA molecule is usually trapped between two contacts (see, e.g., Refs. $[14,18])$. If the trapped molecule is not aligned

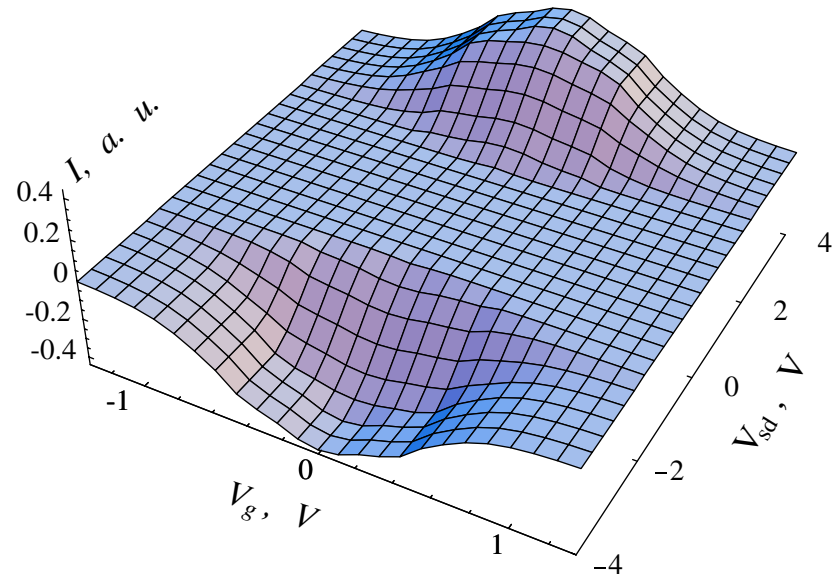

FIG. 2 (color online). Current-voltage characteristics of the DNA molecule within a wide range of source-drain and gate voltages. 


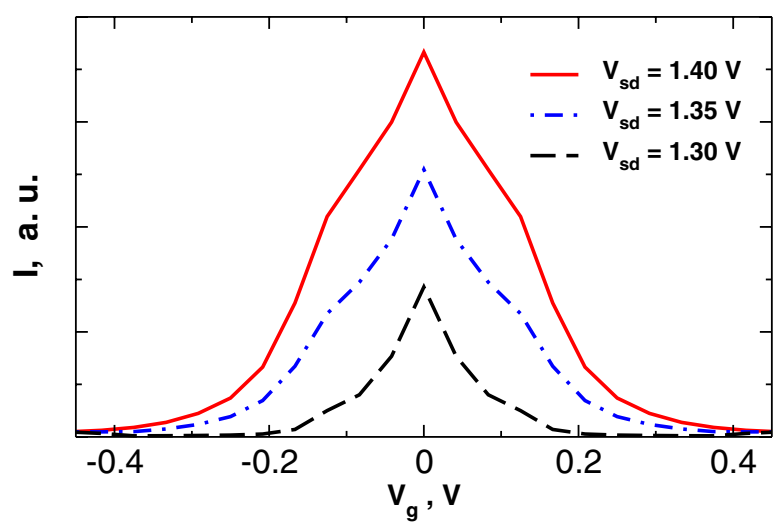

FIG. 3 (color online). The dependence of the DNA sourcedrain current on the gate voltage $V_{g}$ at different fixed sourcedrain voltages $V_{\text {sd }}$, indicated in the plot.

with the intercontact electric field then there exists the component of the field perpendicular to the molecule axis [see the inset of Fig. 4(a)], which produces the gating effect. For such a tilted molecule of length $L$, the gate voltage drop depends on the source-drain voltage:

$$
V_{g}=\frac{2 r}{L} \tan (\alpha) V_{\mathrm{sd}}
$$

Thus, on the one hand, the current tends to increase with the source-drain voltage, while on the other, higher source-

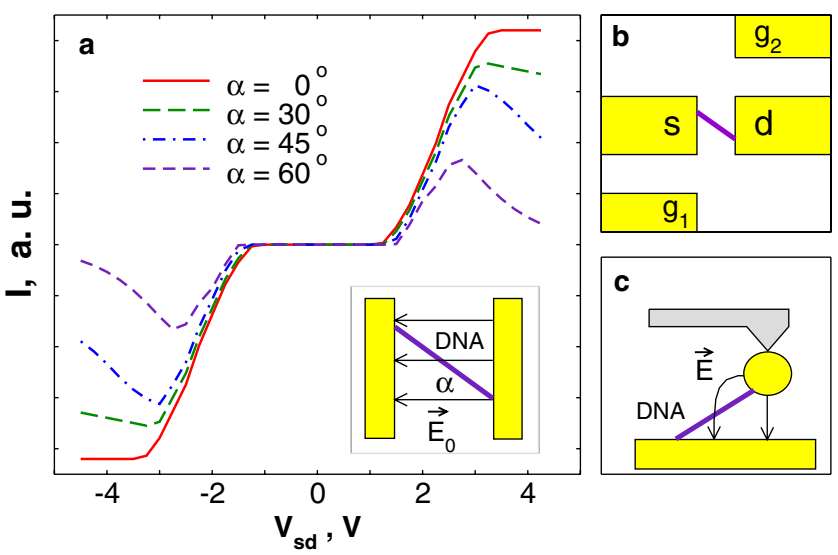

FIG. 4 (color online). (a) Nonmonotonic current-voltage characteristics of the single molecule analog of the Esaki diode for different angles $\alpha$ between the tilted molecule and the intercontact electric field; inset-a scheme of the device. (b),(c) proposed experimental setups to observe the predicted nonmonotonic $I-V$ curves. Panel (b) illustrates the four contact configuration that is put forward for asymmetric electrostatic trapping of a DNA molecule: applying appropriate biases to all four contacts, a tilted electrostatic trapping field can be induced between the electrodes "s" and "d". A polarized molecule aligns with the field and gets trapped between contacts s and $\mathrm{d}$, being tilted with respect to them. Electrodes s and d can then be used for measurements, resulting in the experimental configuration presented in the inset of panel (a). drain voltage leads to stronger gating, which tends to reduce the current. The interplay of the two opposite contributions can lead to new physical effects.

Current-voltage characteristics of a tilted DNA for different angles $\alpha$ between the molecule and the homogeneous interelectrode field are presented in Fig. 4(a). The $I$ - $V$ curves are nonmonotonic and have a region with the negative differential resistance, similar to those of the tunneling diode, which suggests that the proposed device is a single molecule analog of the Esaki diode.

We performed similar calculations for the poly(A)poly(T) DNA, other parameter sets (e.g., ionization energies $[49,50]$, highest occupied molecular orbital/LUMO energies from Ref. [28]), gating electric field profiles, temperatures, and within the framework of the dangling backbone ladder model [42] which accounts for both DNA bases and backbones. The gating effect was found to be generic. We note that the ratio of the DNA length to its fulltwist period, the phase $\varphi_{0}$, and other factors that change the symmetry of the system can result in some qualitative changes. Nevertheless, the strong dependence of the source-drain current on the perpendicular field remains intact.

The underlying gating effect is the direct consequence of the helix geometry of the DNA strands only: the modulation of the strand potential by the gating field [see Eq. (1)] modifies the energy spectrum and reconstructs transmission bands. At the nonzero gating field each band splits into several minibands that are degrading as the field increases; outer minibands degrade faster, which leads to the effective increase of transmission and voltage gaps (see Fig. 1). This mechanism should be taken into account for the correct interpretation of experiments with electrostatically coupled gates.

With a view to observe the predicted effect, the following experimental setups can be proposed. In Ref. [14] a poly $(\mathrm{G})-\operatorname{poly}(\mathrm{C})$ DNA molecule was deposited between two contacts by the electrostatic trapping [51]. If a molecule can deliberately be put at a sufficiently large angle to the contacts then the component of the intercontact field perpendicular to the molecule would produce the sought gating effect as we argue above. During the electrostatic trapping, a DNA molecule becomes polarized by the trapping field and is attracted to the volume where the field is maximum (i.e., to the interelectrode region). The polarized molecule tends to align with the field, thus, asymmetric deposition can be achieved by creating an asymmetric trapping field between the electrodes [see caption of Fig. 4(b) for details].

Another experimental setup was discussed in Ref. [18] where a DNA molecule was trapped between a substrate and a golden nanoparticle suspended from the metalcoated tip of an AFM microscope. It should be possible to keep the DNA molecule tilted with respect to the substrate by appropriate displacements of the tip, in which 
case there would exist a component of the tip-substrate electric field perpendicular to the DNA base stacking direction [see Fig. 4(c)]. We note that the rise of the current that was observed during the retraction of the tip from the surface at constant tip voltage [18] can be explained by the gating effect, which confirms indirectly our prediction. It would therefore be desirable to measure the currentvoltage characteristic of a constantly tilted molecule, which is expected to be nonmonotonic.

In summary, we have demonstrated for the first time that the intrinsic helix conformation of DNA strands has strong impact on transport properties of the molecule. We consider the periodic DNA and show that the electric current through it (in the base stacking direction) can be driven by the perpendicular electric field, suggesting such applications as the field-effect transistor.

We propose also a new molecular device: the periodic DNA trapped between two contacts at an appropriate angle to them (at about $45^{\circ}$ ). The current-voltage characteristic of such a device is nonmonotonic and has a region of the negative differential resistance, analogous to that of the Esaki tunneling diode.

To conclude, the predicted gating effect opens a possibility to use DNA for various novel molecular devices. The same argumentation may also apply to G4-DNA and proteins, many of which have the $\alpha$-helix conformation [52].

The author is grateful to F. Domínguez-Adame, E. Maciá, R. Gutierrez, and V. Malyshev for fruitful discussions, V. M.'s constant encouragement throughout the study and comments on the manuscript are appreciated. This study was supported by MEC under projects Ramón y Cajal and MOSAICO.

[1] M. A. Ratner, Nature (London) 397, 480 (1999).

[2] D. Porath, G. Cuniberti, and R. Di Felice, Top. Curr. Chem. 237, 183 (2004).

[3] R. G. Enders, D. L. Cox, and R. R. P. Singh, Rev. Mod. Phys. 76, 195 (2004).

[4] P. J. Dandliker, R. E. Holmlin, and J. K. Barton, Science 275, 1465 (1997).

[5] M. Mertig et al., Eur. Phys. J. D 9, 45 (1999).

[6] K. Keren et al., Science 302, 1380 (2003).

[7] Introducing Molecular Electronics, edited by G. Cuniberti, G. Fagas, and K. Richter (Springer, Berlin, 2005).

[8] D. B. Tippin, B. Ramakrishnan, and M. Sundaralingam, J. Mol. Biol. 270, 247 (1997).

[9] A. Yu. Kasumov et al., Science 291, 280 (2001).

[10] Y. Okahata et al., J. Am. Chem. Soc. 120, 6165 (1998).

[11] H. W. Fink and C. Schönenberger, Nature (London) 398, 407 (1999).

[12] A. Rakitin et al., Phys. Rev. Lett. 86, 3670 (2001).

[13] O. Legrand, D. Côte, and U. Bockelmann, Phys. Rev. E 73, 031925 (2006).
[14] D. Porath et al., Nature (London) 403, 635 (2000).

[15] K. H. Yoo et al., Phys. Rev. Lett. 87, 198102 (2001).

[16] J. S. Hwang et al., Appl. Phys. Lett. 81, 1134 (2002).

[17] B. Xu et al., Nano Lett. 4, 1105 (2004).

[18] H. Cohen et al., Proc. Natl. Acad. Sci. U.S.A. 102, 11589 (2005).

[19] E. Braun et al., Nature (London) 391, 775 (1998).

[20] A. J. Storm et al., Appl. Phys. Lett. 79, 3881 (2001).

[21] E. Artacho et al., Mol. Phys. 101, 1587 (2003).

[22] P. J. de Pablo et al., Phys. Rev. Lett. 85, 4992 (2000).

[23] R. N. Barnet et al., Science 294, 567 (2001).

[24] E. B. Starikov, Philos. Mag. Lett. 83, 699 (2003).

[25] H. Wang, J. P. Lewis, and O. F. Sankey, Phys. Rev. Lett. 93, 016401 (2004).

[26] A. Hübsch et al., Phys. Rev. Lett. 94, 178102 (2005).

[27] E. B. Starikov, Philos. Mag. 85, 3435 (2005).

[28] H. Mehrez and M. P. Anantram, Phys. Rev. B 71, 115405 (2005).

[29] K. Iguchi, Int. J. Mod. Phys. B 11, 2405 (1997); J. Phys. Soc. Jpn. 70, 593 (2001).

[30] J. Jortner et al., Proc. Natl. Acad. Sci. U.S.A. 95, 12759 (1998).

[31] G. Cuniberti et al., Phys. Rev. B 65, 241314(R) (2002).

[32] S. Roche et al., Phys. Rev. Lett. 91, 228101 (2003).

[33] S. Roche, Phys. Rev. Lett. 91, 108101 (2003).

[34] M. Unge and S. Stafstrom, Nano Lett. 3, 1417 (2003).

[35] M. R. D'Orsogna and R. Bruinsma, Phys. Rev. Lett. 90, 078301 (2003).

[36] K. Iguchi, Int. J. Mod. Phys. B 17, 2565 (2003); 18, 1845 (2004).

[37] H. Yamada, Int. J. Mod. Phys. B 18, 1697 (2004); Phys. Lett. A 332, 65 (2004).

[38] V. Apalkov and T. Chakraborty, Phys. Rev. B 72, 161102(R) (2005).

[39] R. Gutiérrez, S. Mandal, and G. Cuniberti, Nano Lett. 5, 1093 (2005).

[40] S. Kohler, J. Lehmann, and P. Hänggi, Phys. Rep. 406, 379 (2005).

[41] H. Yamada et al., Eur. Phys. J. E 17, 149 (2005).

[42] D. Klotsa, R. A. Römer, and M. S. Turner, Biophys. J. 89, 2187 (2005).

[43] R. Gutiérrez et al., Phys. Rev. B 74, 235105 (2006).

[44] E. Maciá and S. Roche, Nanotechnology 17, 3002 (2006).

[45] C. S. Lent and D. J. Kirkner, J. Appl. Phys. 67, 6353 (1990).

[46] D. Z.-Y. Ting, E. T. Yu, and T. C. McGill, Phys. Rev. B 45, 3583 (1992).

[47] D. K. Ferry and S. M. Goodnick, Transport in Nanostructures (Cambridge University Press, New York, 1997).

[48] M. S. Xu et al., Appl. Phys. Lett. 87, 083902 (2005).

[49] H. Sugiyama and I. Saito, J. Am. Chem. Soc. 118, 7063 (1996).

[50] A. Voityuk et al., J. Chem. Phys. 114, 5614 (2001).

[51] A. Bezryadin, C. Dekker, and G. Schmid, Appl. Phys. Lett. 71, 1273 (1997).

[52] C. Branden and J. Tooze, Introduction to Protein Structure (Garland, New York, 1999), 2nd ed. 